\title{
Ombro do arremessador: Artigo de atualização*
}

\section{Pitcher Shoulder: Update Article}

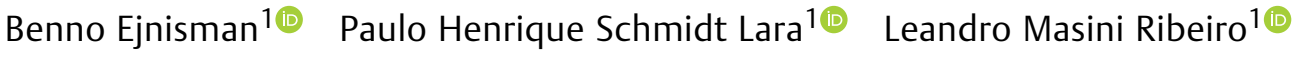 \\ Paulo Santoro Belangero ${ }^{1}{ }^{\circledR}$
} ${ }^{1}$ Grupo de Ombro e Cotovelo, Centro de Traumatologia do Esporte,
Escola Paulista de Medicina, Universidade Federal de São Paulo, São
Paulo, SP, Brasil

Rev Bras Ortop 2021;56(3):275-280.

\begin{abstract}
Endereço para correspondência Paulo Henrique Schmidt Lara, MD Centro de Traumatologia do Esporte, Escola Paulista de Medicina, Universidade Federal de São Paulo, Rua Estado de Israel, 636, Vila Clementino, São Paulo, SP, 04022-001, Brasil

(e-mail: phslara@gmail.com; bennoale@uol.com.br).
\end{abstract}

\section{Resumo \\ Palavras-chave \\ - ombro \\ - instabilidade articular \\ - traumatismos em atletas}

\section{Abstract \\ Keywords \\ - shoulder \\ - joint instability \\ - athletic injuries}

A maioria das lesões do ombro ocorre devido aos movimentos repetitivos acima do nível da cabeça. Antes de estudar o tratamento dessas lesões, é fundamental que os profissionais de saúde tenham um entendimento da etiologia e dos mecanismos que causam essas patologias. O ato do arremesso acima do nível da cabeça exige considerável coordenação de todo o corpo, desde o momento de geração de força até o final do arremesso. O ombro é um componente crucial da cadeia cinética da extremidade superior, por transmitir a força gerada na extremidade inferior para o braço e mão para produzir velocidade e precisão no lançamento da bola.

Most shoulder injuries occur due to repetitive overhead movements. Before studying the treatment of these shoulder injuries, it is paramount that health professionals have an understanding of the etiology of and the underlying mechanisms for shoulder pathologies. The act of overhead throwing is an eloquent full-body motion that requires tremendous coordination from the time of force generation to the end of the pitch. The shoulder is a crucial component of the upper-body kinetic chain, as it transmits force created in the lower body to the arm and hand to provide velocity and accuracy to the pitch.

\section{Introdução}

Atletas arremessadores têm uma tendência a desenvolver lesões no ombro como resultado das forças intensas a que essa articulação é submetida durante o arremesso. Os estabilizadores dinâmicos da articulação glenoumeral incluem o

Trabalho desenvolvido no Grupo de Ombro e Cotovelo, Centro de Traumatologia do Esporte, Escola Paulista de Medicina, Universidade Federal de São Paulo, São Paulo, SP, Brasil.

recebido

05 de Julho de 2019

aceito

05 de Dezembro de 2019

Publicado on-line

Março 23, 2020 manguito rotador, músculos escapulotorácicos, e o tendão da cabeça longa do bíceps, e os estabilizadores estáticos incluem a anatomia óssea, o lábio fibrocartilaginoso, e a cápsula articular. Um evento traumático é o bastante para causar a lesão; no entanto, é a sobrecarga repetitiva que mais comumente leva à falha de uma ou mais dessas estruturas. $\mathrm{O}$ ato do arremesso exige uma ação coordenada que progride da ponta dos dedos do pé até os dedos da mão. Essa sequência de eventos foi descrita conceitualmente como cadeia cinética. ${ }^{1}$

(C) 2020. Sociedade Brasileira de Ortopedia e Traumatologia. All rights reserved.

This is an open access article published by Thieme under the terms of the Creative Commons Attribution-NonDerivative-NonCommercial-License, permitting copying and reproduction so long as the original work is given appropriate credit. Contents may not be used for commercial purposes, or adapted, remixed, transformed or built upon. (https://creativecommons.org/ licenses/by-nc-nd/4.0/)

Thieme Revinter Publicações Ltda., Rua do Matoso 170, Rio de Janeiro, RJ, CEP 20270-135, Brazil 
Para esta cadeia funcionar efetivamente, uma atividade muscular sequencial é necessária para que a energia gerada na parte inferior do corpo seja transmitida à parte superior e finalmente até a bola. ${ }^{2}$ A velocidade da bola é determinada pela eficiência deste processo. Rotação corporal e posicionamento da escápula são elementos-chave na cadeia cinemática. Em arremessadores profissionais, há um delicado balanço entre mobilidade do ombro e estabilidade. O ombro precisa ser móvel o suficiente para que os extremos de rotação sejam alcançados, e para que a velocidade seja transmitida à bola; mas, ao mesmo tempo, o ombro deve permanecer estável para que a cabeça umeral permaneça dentro da cavidade glenoidal, criando um fulcro estável para rotação, o que é conhecido como "paradoxo do arremessador". ${ }^{3}$ A cada arremesso, o envelope de partes moles que circunda o ombro é submetido a uma carga que é muito próxima da carga máxima suportada, o que acarreta uma propensão a lesões. ${ }^{3}$ Enquanto os padrões de lesão dos ombros de arremessadores são comuns e previsíveis, ainda há controvérsia sobre os mecanismos exatos que levam a essas lesões. Estudos recentes biomecânicos têm ajudado a melhorar o entendimento da patogênese das lesões nos atletas arremessadores. ${ }^{4-6}$ Além disso, dados quantitativos sobre biomecânica e cinemática, tanto normal quanto patológica, têm ajudado no desenvolvimento de estratégias para a prevenção de lesões, o tratamento e a reabilitação. ${ }^{7-9}$

\section{Cinemática do Arremesso}

$\mathrm{O}$ arremesso foi dividido em seis fases, que geralmente levam menos de dois segundos para ocorrer. ${ }^{10,11}$ As três primeiras fases consistem de preparação, passada e elevação do braço, e levam aproximadamente 1,5 segundo no total. Embora a quarta fase, a aceleração, dure cerca de 0,05 segundo, as maiores velocidades angulares e a maior mudança na rotação ocorrem nela. ${ }^{12}$ As duas últimas fases são a desaceleração e a execução, e, juntas, duram aproximadamente 0,35 segundo ${ }^{12}$

(-Figura 1). Como certas lesões ocorrem em certas fases, é importante determinar quando a dor ou um problema ocorrem.

A velocidade da bola depende de uma variedade de fatores biomecânicos, mas está mais diretamente relacionada à quantidade de rotação lateral que o ombro alcança. ${ }^{13}$ Para gerar a máxima velocidade de arremesso da maneira mais eficiente, as extremidades inferior e superior devem trabalhar de forma sincronizada e coordenada. Arremessadores profissionais podem gerar velocidades de bola que excedem $144,8 \mathrm{~km} / \mathrm{h}$; para criar uma velocidade dessas, o ombro alcança velocidades angulares de até $7 \mathrm{mil}$ graus/s. ${ }^{13}$ Após a soltura da bola, o ombro de um arremessador profissional pode ser exposto a forças distrativas de até $950 \mathrm{~N} .{ }^{14} \mathrm{Na}$ fase de desaceleração, as forças de compressão criadas pelos músculos do manguito rotador e do deltoide atingem a faixa de $1.090 \mathrm{~N}$, e as forças de cisalhamento posterior, até $400 \mathrm{~N} .{ }^{14} \mathrm{~A}$ parte anterior da cápsula resiste a aproximadamente $800 \mathrm{~N}$ a $1200 \mathrm{~N}$ em indivíduos de 20 a 30 anos. $^{15}$ Portanto, se as forças compressivas não contrabalancearem as forças intensas de distração, lesões ocorrerão. ${ }^{15} \mathrm{O}$ estudo de Kibler et al ${ }^{1}$ contribuíram bastante para o entendimento sobre a dinâmica escapular, a prevenção de lesões, e o tratamento. Estima-se que apenas metade da energia cinética transmitida à bola vem do braço e do ombro. $\mathrm{A}$ outra metade é gerada pela rotação do tronco e dos membros inferiores, e é transferida para a extremidade superior por meio da articulação escapulotorácica, fazendo desta articulação uma importante, porém frequentemente negligenciada, parte da cadeia cinética. ${ }^{16} \mathrm{~A}$ análise dinâmica dos ombros durante o arremesso contribuiu para o nosso atual conhecimento da função normal e anormal, e, ao demonstrar quais grupos musculares estão ativos durante o arremesso durante cada fase, ajudou a orientar o desenvolvimento de programas de prevenção e reabilitação. ${ }^{17}$

\section{Patogênese das Lesões}

O ombro do arremessador está suscetível a lesões devido à convergência dos seguintes fatores: atenuação dos constritores da cápsula anterior, contratura da cápsula posterior, desenvolvimento de discinesia escapular, quebra da cadeia cinética, e contato repetitivo do tubérculo maior e do lábio posterossuperior. Cada um desses fatores foi avaliado, e estratégias foram sugeridas para a prevenção de lesão.

\section{Frouxidão da Cápsula Anterior}

Estudos biomecânicos demonstraram que a cápsula anterior, particularmente a banda anterior do ligamento glenoumeral inferior, é a principal restritora da translação anterior do úmero com o braço em abdução e rotação lateral. ${ }^{18-20}$ Portanto, estresse repetitivo nessa área e o desejo do arremessador de atingir níveis cada vez maiores de rotação
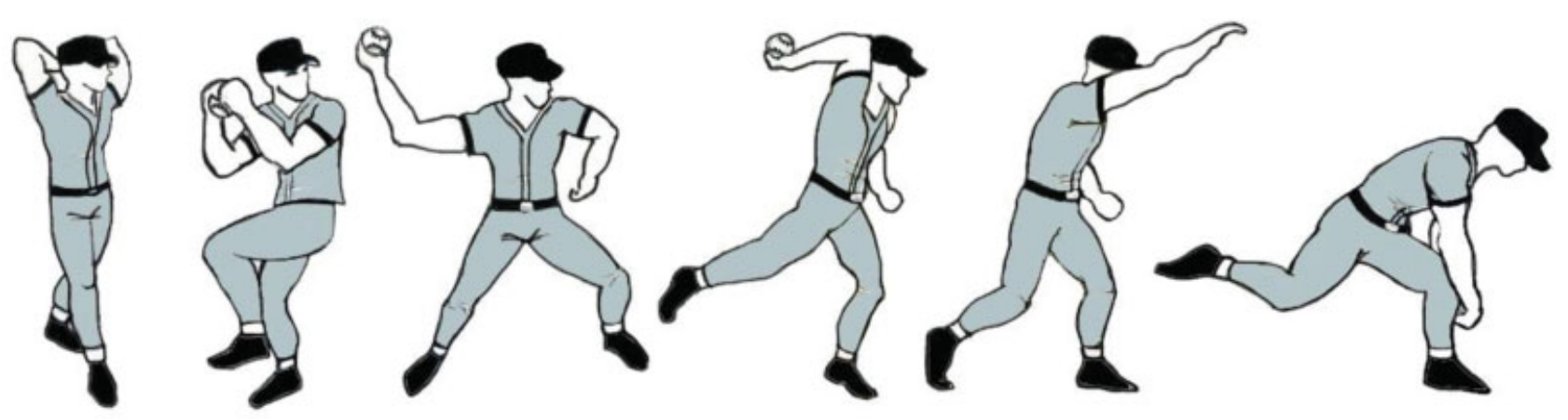

Fig. 1 Fases do arremesso: (1) preparação; (2) passada; (3) elevação do braço; (4) aceleração; (5) desaceleração; e (6) execução/finalização. Fonte: Desenho do autor. 
lateral levam a uma frouxidão da cápsula anterior. ${ }^{18-20}$ Embora as causas atribuídas sejam controversas, arremessadores de fato apresentam mais rotação lateral passiva do que rotação do ombro contralateral. ${ }^{21,22}$ Se o ganho de rotação lateral for maior do que a perda de rotação medial, ocorre a frouxidão dos restritores. ${ }^{21,22}$ Defendendo esse argumento, 0 trabalho de Jobe et $\mathrm{al}^{23}$ descreve o tensionamento da cápsula anterior como um meio de retorno do atleta à atividade de arremesso. Embora este procedimento tenha sido bemsucedido para os pacientes do estudo de Jobe et $\mathrm{al}^{23}$ no qual $68 \%$ dos pacientes apresentaram resultados excelentes e retornaram ao nível pré-lesão, e 96\% ficaram satisfeitos com a cirurgia, a violação do subescapular e o tensionamento excessivo explicam por que nem todos os pacientes conseguiram retornar ao nível pré-lesão após a reconstrução. Com a progressão da frouxidão anterior, há aumento da rotação lateral e aumento do contato entre a parte posterior do manguito e o lábio, o que facilita a ocorrência de lesão. ${ }^{24}$

\section{Contratura da Cápsula Posterior}

Com o tempo, os arremessadores apresentam rotação medial diminuída, principalmente quando medida na posição de abdução. ${ }^{25}$ Acredita-se que essa diminuição de rotação medial ocorra por duas razões. Primeiramente, o aumento da retroversão do úmero observada em arremessadores manifesta-se com uma perda de rotação medial. Entretanto, esta perda, devido à remodelação óssea, é acompanhada por ganho simétrico de rotação lateral. ${ }^{25}$ Outro meio de perda de rotação medial é a contratura da cápsula posterior. Acredita-se que o déficit de rotação medial da articulação glenoumeral ocorra como um processo cicatricial em resposta às forças distrativas crônicas aplicadas à cápsula posterior na fase de execução do arremesso. ${ }^{25}$ Perda rotacional devido a contratura capsular é evidente quando o déficit de rotação medial da articulação glenoumeral excede aquele que pode ser explicado apenas pelo remodelamento ósseo (mais do que $12^{\circ}$ ), e quando a perda de rotação medial excede o aumento de rotação lateral comparado ao lado contralateral. ${ }^{25}$

\section{Consequências Biomecânicas do Déficit de Rotação Medial da Articulação Glenoumeral}

Estudos clínicos e biomecânicos atuais ${ }^{26,27}$ mostraram que o déficit de rotação medial da articulação glenoumeral pode ser o evento sentinela na cascata patológica pela qual muitos arremessadores passam. Os autores verificaram que arremessadores que apresentavam lesões labiais superiores apresentavam déficit de rotação medial da articulação glenoumeral acima de $25^{\circ} .^{26,27}$ Mesmo pequenos graus de déficit de rotação medial da articulação glenoumeral (como $5^{\circ}$, por exemplo) colocam o ombro em risco de lesão e eventual necessidade de cirurgia. ${ }^{26,27} 0$ deslocamento posterossuperior que ocorre com o déficit de rotação medial da articulação glenoumeral é devido à contratura capsular posterior e inferior, o que não permite a rotação lateral total do úmero. Com isso, o atleta começa a fazer a rotação em volta de um novo centro de rotação, que é mais posterior e proximal. Essencialmente, uma cápsula posteroinferior que

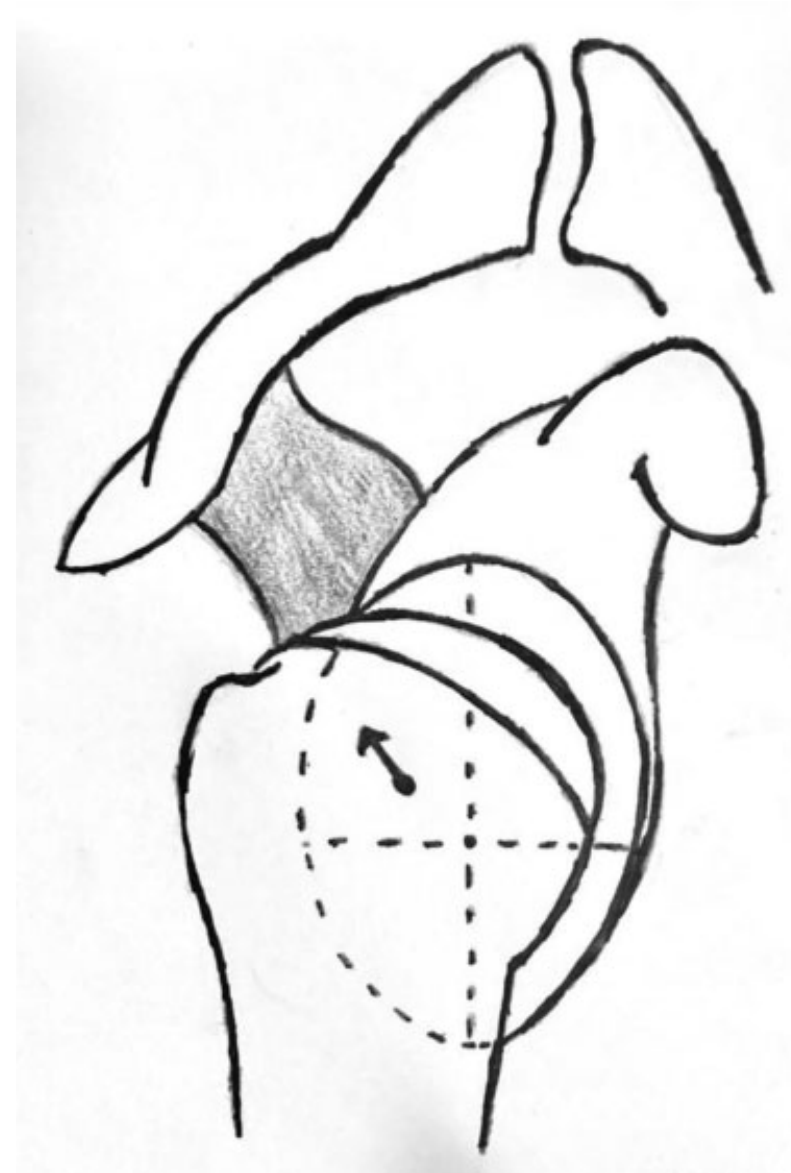

Fig. 2 Deslocamento posterior e proximal da cabeça umeral. Fonte: Desenho do autor.

sofreu contratura desloca o úmero mais posterior e proximalmente (-Figura 2). ${ }^{6}$

\section{Discinesia Escapular}

A discinesia é uma anormalidade estática ou dinâmica da posição escapular. Dores no ombro levam a uma inibição dos músculos trapézio inferior e serrátil anterior, e contratura do trapézio superior e do peitoral menor. ${ }^{28-31}$ Este desequilíbrio muscular leva a uma protração da escápula. Arremessadores com perda de rotação medial devido a contratura capsular acabam usando a rotação medial escapular para realizar o arremesso. Com o passar do tempo, a escápula perde os restritores estáticos e provavelmente sobrecarrega os restritores dinâmicos, e, com isso, a escápula desvia-se da linha média e desloca-se anteriormente. ${ }^{32}$ Thomas et al $^{33}$ demonstraram que quanto maior o déficit de rotação medial, maior as alterações da posição e mobilidade da escápula. Avaliaram 43 jogadores profissionais de beisebol, e em 22 atletas foram encontrados déficits maiores do que $15^{\circ}$, nos quais havia maior discinesia escapular, com significância estatística. Em outro estudo de Thomas et $\mathrm{al}^{34}$ foi demonstrada uma relação temporal entre a discinesia escapular e o déficit de rotação medial da articulação glenoumeral, no qual jogadores de beisebol com mais anos de prática demonstraram maior déficit, com significância estatística. 


\section{Efeitos da Protração Escapular Excessiva}

Há diversas consequências biomecânicas de uma escápula com protração ou rotação medial excessivas. Primeiramente, há uma fraqueza do manguito rotador. Como o complexo do manguito rotador essencialmente origina-se da escápula, se houver uma plataforma instável, não há uma função adequada destes músculos. ${ }^{35}$ Além disso, a maior protração aumenta a versão da escápula, levando a uma desestabilização anterior e a aumento da sobrecarga nos ligamentos anteriores. ${ }^{36} \mathrm{~A}$ protração excessiva também aumenta o grau de impacto entre o manguito rotador posterior e a região posterossuperior da glenoide durante abdução e rotação lateral. ${ }^{26} \mathrm{O}$ estudo de Laudner et al $^{37}$ avaliou que atletas arremessadores diagnosticados com impacto interno patológico apresentaram aumento com significância estatística da elevação da articulação esternoclavicular e desvio escapular posterior durante elevação do ombro no plano da escápula.

\section{Condições Patológicas Comuns e Opções de Tratamento}

\section{Mobilidade e Instabilidade}

Mobilidade é definida como movimentação passiva de uma articulação em uma direção especial ou rotação. ${ }^{38,39}$ Hiperelasticidade pode ser fisiológica ou patológica, e pode predispor a lesões. Já o termo instabilidade do ombro é reservado para a sensação de um movimento excessivo da cabeça umeral em relação à glenoide, que geralmente está associada a dor ou desconforto. Poucos arremessadores apresentam sintomas de instabilidade, embora o termo instabilidade tenha sido usado em muitos estudos para descrever a síndrome que ocorre em arremessadores. Enquanto algum grau de hiperelasticidade possa auxiliar 0 atleta a competir em alto nível em esportes que envolvem arremesso, o seu excesso pode ser responsável pelo desenvolvimento de certas condições patológicas do ombro. Isso tem sido chamado de instabilidade atraumática, que se acredita que ocorre pelo estresse repetitivo durante os arremessos. ${ }^{40}$ Kuhn et al ${ }^{41}$ cunharam o termo hiperelasticidade patológica, que nós também acreditamos ser uma descrição mais precisa do que está realmente acontecendo.

\section{Lesões SLAP}

A lesão do lábio superior de anterior para posterior (superior labral tear from anterior to posterior, SLAP, em inglês) é uma causa clínica importante de dor no ombro. Burkhart e Mor$\operatorname{gan}^{42}$ propuseram que as lesões SLAP em arremessadores ocorrem pelo mecanismo de "peel-back," que é definido como um aumento da tensão na origem do bíceps durante a máxima rotação lateral durante o arremesso. Estudos em laboratório mostraram que a cabeça longa do bíceps é um restritor dinâmico importante da rotação lateral quando o braço é abduzido. ${ }^{43}$ Inicialmente, preconiza-se o tratamento conservador, cujos principais objetivos são a diminuição da dor, o ganho de arco de movimento, e a concentração em fortalecimento dinâmico com ênfase nos estabilizadores da escápula e do manguito rotador. ${ }^{42} \mathrm{Na}$ falha deste, indica-se o tratamento cirúrgico, que geralmente é artroscópico, e varia de acordo com o grau da lesão. ${ }^{42}$

\section{Lesões do Manguito Rotador}

Cerca de $62 \%$ das lesões do manguito rotador dos arremessadores são lesões parciais articulares. ${ }^{6}$ Estas lesões em arremessadores são encontradas geralmente posterossuperiormente na junção das inserções dos músculos supra e infraespinais. ${ }^{44,45}$ Fisioterapia deve ser considerada o tratamento inicial das lesões parciais do manguito em arremessadores. 0 simples desbridamento não tem demonstrado bons resultados em arremessadores. $\mathrm{O}$ estudo de Payne et $\mathrm{a}^{46}$ avaliou atletas submetidos ao desbridamento simples que foram divididos em dois grupos (arremessadores com lesões traumáticas e lesões não traumáticas). Em pacientes com lesões traumáticas, houve resultado satisfatório em $86 \%$ dos casos e $64 \%$ retornaram ao esporte. Já nos atletas com lesões não traumáticas, houve resultado satisfatório em $66 \%$ dos casos, e retorno ao esporte em $45 \%$ deles.

\section{Impacto}

Diferentes tipos de impactos foram descritos na literatura, incluindo o impacto clássico, o subacromial, o secundário, e o interno. ${ }^{47-52} \mathrm{O}$ impacto interno é um fenômeno patológico no qual o manguito rotador encontra-se com o aspecto posterossuperior do lábio com o ombro no grau máximo de abdução e rotação lateral. ${ }^{53,54}$ Vários estudos demonstraram que esse tipo de impacto é mais provavelmente causado pela fadiga da musculatura da cintura escapular devido à falta de condicionamento ou ao excesso de treinamento. ${ }^{55,56}$ Estes estudos demonstraram que, durante a fase de aceleração do arremesso, o úmero deve estar alinhado com o plano da escápula. A partir do momento em que os músculos ficam fadigados, o úmero sai do plano da escápula, o que é chamado de hiperangulação, que acarreta a sobrecarga da cápsula anterior. ${ }^{57}$

\section{Linhas Gerais do Tratamento}

$\mathrm{O}$ tratamento se inicia com medidas conservadoras. A contratura da cápsula posterior deve ser abordada, e deve ser realizado um programa de alongamento e mobilização. Esses alongamentos devem isolar a articulação glenoumeral para que a compensação escapular seja minimizada. ${ }^{26} \mathrm{~A}$ avaliação da cadeia cinética é essencial. Contratura lombar, fraqueza dos abdutores do quadril, e diminuição da rotação medial da perna devem ser investigadas. ${ }^{26} \mathrm{~A}$ discinesia escapular, a qual geralmente está presente, pode geralmente ser tratada com exercícios que auxiliem na restauração da mobilidade normal escapular. 0 primeiro passo da reabilitação escapular deve focar na reeducação neuromuscular dos músculos estabilizadores escapulares. Fortalecimento deve ser iniciado após esta fase. ${ }^{26} \mathrm{O}$ fortalecimento dos músculos do manguito rotador deve ser feito, especialmente do infraespinal, por meio de exercícios de rotação lateral com resistência, o que protege o manguito rotador de lesões. ${ }^{26}$

O tratamento cirúrgico é indicado em casos de falha do tratamento conservador. Tenta-se geralmente pelo menos de três a quatro meses de fisioterapia, a qual pode ser prolongada caso o atleta apresente melhora progressiva do quadro. ${ }^{26} \mathrm{~A}$ maioria dos arremessadores, especialmente os mais jovens, são capazes de recuperação a partir do momento em que há resolução da discinesia escapular e do déficit de rotação medial. 


\section{Considerações Finais}

O desempenho de atletas arremessadores é frequentemente limitado por lesões no ombro. Esses problemas são complexos e, portanto, de difícil manejo. Os problemas ocorrem como resultado de uma combinação de desquilíbrio muscular, fadiga muscular, hiperfrouxidão da cápsula anterior, contratura da cápsula posterior, mecânica alterada do arremesso, discinesia escapular, aumento da retroversão umeral, e microtraumas repetitivos. Como resultado, em arremessadores observamos lesões que envolvem o lábio, o lado articular da parte posterior do manguito rotador, e a inserção proximal da cabeça longa do bíceps.

Os mecanismos e etiologias das lesões em arremessadores estão se tornando mais bem definidos. Embora haja controvérsia sobre qual seria o evento inicial, os padrões típicos de lesão continuam os mesmos.

Antes de pensarmos sobre opções de tratamento, é fundamental obter uma história detalhada, exame físico, e estudos adicionais de imagem para chegar ao diagnóstico correto. $\mathrm{O}$ tratamento de lesões do ombro deve ser iniciado com um protocolo que centrado no restabelecimento do arco de movimento, no fortalecimento e no alongamento específico para promover a estabilidade da escápula, do ombro, e dos músculos profundos da região abdominal, lombar e pélvica que têm como finalidade manter a estabilidade dessa região. Além disso, os médicos, fisioterapeutas e preparadores físicos envolvidos com atletas arremessadores devem ter ampla compreensão de toda a cascata fisiopatológica que leva a lesões nesses atletas.

\section{Conflito de Interesses}

Os autores declaram não haver conflito de interesses.

\section{Referências}

1 Kibler WB. The role of the scapula in athletic shoulder function. Am J Sports Med 1998;26(02):325-337

2 Hirashima M, Kadota H, Sakurai S, Kudo K, Ohtsuki T. Sequential muscle activity and its functional role in the upper extremity and trunk during overarm throwing. J Sports Sci 2002;20(04):301-310

3 Wilk KE, Meister K, Andrews JR. Current concepts in the rehabilitation of the overhead throwing athlete. Am J Sports Med 2002; 30(01):136-151

4 Werner SL, Guido JA Jr, Stewart GW, McNeice RP, VanDyke T, Jones DG. Relationships between throwing mechanics and shoulder distraction in collegiate baseball pitchers. J Shoulder Elbow Surg 2007;16(01):37-42

5 Bakshi N, Freehill MT. The Overhead Athletes Shoulder. Sports Med Arthrosc Rev 2018;26(03):88-94

6 Burkhart SS, Morgan CD, Kibler WB. The disabled throwing shoulder: spectrum of pathology Part I: pathoanatomy and biomechanics. Arthroscopy 2003;19(04):404-420

7 Mlynarek RA, Lee S, Bedi A. Shoulder Injuries in the Overhead Throwing Athlete. Hand Clin 2017;33(01):19-34

8 Kuhn JE. Throwing, the Shoulder, and Human Evolution. Am J Orthop 2016;45(03):110-114

9 Zaremski JL, Wasser JG, Vincent HK. Mechanisms and Treatments for Shoulder Injuries in Overhead Throwing Athletes. Curr Sports Med Rep 2017;16(03):179-188

10 Meister K. Injuries to the shoulder in the throwing athlete. Part one: Biomechanics/pathophysiology/classification of injury. Am J Sports Med 2000;28(02):265-275
11 Lin DJ, Wong TT, Kazam JK. Shoulder Injuries in the OverheadThrowing Athlete: Epidemiology, Mechanisms of Injury, and Imaging Findings. Radiology 2018;286(02):370-387

12 Pappas AM, Zawacki RM, Sullivan TJ. Biomechanics of baseball pitching. A preliminary report. Am J Sports Med 1985;13(04):216-222

13 Dillman CJ, Fleisig GS, Andrews JR. Biomechanics of pitching with emphasis upon shoulder kinematics. J Orthop Sports Phys Ther 1993;18(02):402-408

14 Kuhn JE, Lindholm SR, Huston LJ. Failure of the biceps-superior labral complex (SLAP lesion) in the throwing athlete: a biomechanical model comparing maximal cocking to early deceleration. [abstract]J Shoulder Elbow Surg 2000;9:463

15 Reeves B. Experiments on the tensile strength of the anterior capsular structures of the shoulder in man. J Bone Joint Surg Br 1968;50(04):858-865

16 Chu SK, Jayabalan P, Kibler WB, Press J. The Kinetic Chain Revisited: New Concepts on Throwing Mechanics and Injury. PM R 2016;8(3, Suppl)S69-S77

17 David G, Magarey ME, Jones MA, Dvir Z, Türker KS, Sharpe M. EMG and strength correlates of selected shoulder muscles during rotations of the glenohumeral joint. Clin Biomech (Bristol, Avon) 2000;15(02):95-102

18 O'Connell PW, Nuber GW, Mileski RA, Lautenschlager E. The contribution of the glenohumeral ligaments to anterior stability of the shoulder joint. Am J Sports Med 1990;18(06): 579-584

19 O'Brien SJ, Schwartz RS, Warren RF, Torzilli PA. Capsular restraints to anterior-posterior motion of the abducted shoulder: a biomechanical study. J Shoulder Elbow Surg 1995;4(04):298-308

20 McMahon PJ, Tibone JE, Cawley PW, et al. The anterior band of the inferior glenohumeral ligament: biomechanical properties from tensile testing in the position of apprehension. J Shoulder Elbow Surg 1998;7(05):467-471

21 Bigliani LU, Codd TP, Connor PM, Levine WN, Littlefield MA, Hershon SJ. Shoulder motion and laxity in the professional baseball player. Am J Sports Med 1997;25(05):609-613

22 Brown LP, Niehues SL, Harrah A, Yavorsky P, Hirshman HP. Upper extremity range of motion and isokinetic strength of the internal and external shoulder rotators in major league baseball players. Am J Sports Med 1988;16(06):577-585

23 Jobe FW, Giangarra CE, Kvitne RS, Glousman RE. Anterior capsulolabral reconstruction of the shoulder in athletes in overhand sports. Am J Sports Med 1991;19(05):428-434

24 Karduna AR, McClure PW, Michener LA, Sennett B. Dynamic measurements of three-dimensional scapular kinematics: a validation study. J Biomech Eng 2001;123(02):184-190

25 Kay J, Kirsch JM, Bakshi N, et al. Humeral Retroversion and Capsule Thickening in the Overhead Throwing Athlete: A Systematic Review. Arthroscopy 2018;34(04):1308-1318

26 Burkhart SS, Morgan CD, Kibler WB. Shoulder injuries in overhead athletes. The "dead arm" revisited. Clin Sports Med 2000;19(01): $125-158$

27 Johnson JE, Fullmer JA, Nielsen CM, Johnson JK, Moorman CT 3rd. Glenohumeral Internal Rotation Deficit and Injuries: A Systematic Review and Meta-analysis. Orthop J Sports Med 2018;6(05): 2325967118773322

28 Kibler WB, McMullen J. Scapular dyskinesis and its relation to shoulder pain. J Am Acad Orthop Surg 2003;11(02):142-151

29 Pink MM, Perry J. Biomechanics of the shoulder. St Louis: MosbyYear Book; 1996

30 McClure PW, Michener LA, Sennett BJ, Karduna AR. Direct 3dimensional measurement of scapular kinematics during dynamic movements in vivo. J Shoulder Elbow Surg 2001;10(03): 269-277

31 McQuade KJ, Dawson J, Smidt GL. Scapulothoracic muscle fatigue associated with alterations in scapulohumeral rhythm kinematics during maximum resistive shoulder elevation. J Orthop Sports Phys Ther 1998;28(02):74-80 
32 Pink MM. Understanding the linkage system of the upper extremity. Sports Med Arthrosc Rev 2001;9(01):52-60

33 Thomas SJ, Swanik KA, Swanik CB, Kelly JD 4th. Internal rotation deficits affect scapular positioning in baseball players. Clin Orthop Relat Res 2010;468(06):1551-1557

34 Thomas SJ, Swanik KA, Swanik CB, Kelly JD. Internal rotation and scapular position differences: a comparison of collegiate and high school baseball players. J Athl Train 2010;45(01):44-50

35 Kibler WB, Sciascia A, Dome D. Evaluation of apparent and absolute supraspinatus strength in patients with shoulder injury using the scapular retraction test. Am J Sports Med 2006;34(10): 1643-1647

36 Weiser WM, Lee TQ McMaster WC, McMahon PJ. Effects of simulated scapular protraction on anterior glenohumeral stability. Am J Sports Med 1999;27(06):801-805

37 Laudner KG, Myers JB, Pasquale MR, Bradley JP, Lephart SM. Scapular dysfunction in throwers with pathologic internal impingement. J Orthop Sports Phys Ther 2006;36(07):485-494

38 Wilk KE, Andrews JR, Arrigo CA, Keirns MA, Erber DJ. The strength characteristics of internal and external rotator muscles in professional baseball pitchers. Am J Sports Med 1993;21(01):61-66

39 Ryu RKN, Dunbar WH V, Kuhn JE, McFarland EG, Chronopoulos E, Kim TK. Comprehensive evaluation and treatment of the shoulder in the throwing athlete. Arthroscopy 2002;18(09, Suppl 2):70-89

40 DeFroda SF, Goyal D, Patel N, Gupta N, Mulcahey MK. Shoulder Instability in the Overhead Athlete. Curr Sports Med Rep 2018;17 (09):308-314

41 Kuhn JE, Lindholm SR, Huston LJ, Soslowsky LJ, Blasier RB. Failure of the biceps superior labral complex: a cadaveric biomechanical investigation comparing the late cocking and early deceleration positions of throwing. Arthroscopy 2003;19(04):373-379

42 Burkhart SS, Morgan CD. The peel-back mechanism: its role in producing and extending posterior type II SLAP lesions and its effect on SLAP repair rehabilitation. Arthroscopy 1998;14(06):637-640

43 Kuhn JE, Huston LJ, Soslowsky LJ, Shyr Y, Blasier RB. External rotation of the glenohumeral joint: ligament restraints and muscle effects in the neutral and abducted positions. J Shoulder Elbow Surg 2005;14(1, Suppl S)39S-48S

44 Walch G, Boileau P, Noel E, Donell ST. Impingement of the deep surface of the supraspinatus tendon on the posterosuperior glenoid rim: An arthroscopic study. J Shoulder Elbow Surg 1992;1(05):238-245

45 Miniaci A, Mascia AT, Salonen DC, Becker EJ. Magnetic resonance imaging of the shoulder in asymptomatic professional baseball pitchers. Am J Sports Med 2002;30(01):66-73

46 Payne LZ, Altchek DW, Craig EV, Warren RF. Arthroscopic treatment of partial rotator cuff tears in young athletes. A preliminary report. Am J Sports Med 1997;25(03):299-305

47 Jobe FW, Kvitne RS, Giangarra CE. Shoulder pain in the overhand or throwing athlete. The relationship of anterior instability and rotator cuff impingement. Orthop Rev 1989;18(09):963-975

48 Jobe FW, Jobe CM. Painful athletic injuries of the shoulder. Clin Orthop Relat Res 1983;(173):117-124

49 Warner JJ, Micheli LJ, Arslanian LE, Kennedy J, Kennedy R. Scapulothoracic motion in normal shoulders and shoulders with glenohumeral instability and impingement syndrome. A study using Moiré topographic analysis. Clin Orthop Relat Res 1992;(285):191-199

50 Morris AD, Kemp GJ, Frostick SP. Shoulder electromyography in multidirectional instability. J Shoulder Elbow Surg 2004;13(01): 24-29

51 Crockett HC, Gross LB, Wilk KE, et al. Osseous adaptation and range of motion at the glenohumeral joint in professional baseball pitchers. Am J Sports Med 2002;30(01):20-26

52 Bach HG, Goldberg BA. Posterior capsular contracture of the shoulder. J Am Acad Orthop Surg 2006;14(05):265-277

53 Jobe CM. Posterior superior glenoid impingement: expanded spectrum. Arthroscopy 1995;11(05):530-536

54 Walch G, Liotard JP, Boileau P, Noël E. [Postero-superior glenoid impingement. Another shoulder impingement]. Rev Chir Orthop Repar Appar Mot 1991;77(08):571-574

55 Jobe CM. Superior glenoid impingement. Orthop Clin North Am 1997;28(02):137-143

56 Paley KJ, Jobe FW, Pink MM, Kvitne RS, ElAttrache NS. Arthroscopic findings in the overhand throwing athlete: evidence for posterior internal impingement of the rotator cuff. Arthroscopy 2000;16(01):35-40

57 Jobe CM, Pink MM, Jobe FW, Shaffer B. Anterior shoulder instability, impingement, and rotator cuff tear: theories and concepts. In: Jobe FW, editor. Operative techniques in upper extremity sports injuries. St. Louis, MO: Mosby; 1996:164-176 